\title{
Connaissances et perceptions locales de la dynamique des cuirasses ferrugineuses : Etude de cas en zone Nord-soudanienne et Sud-soudanienne du Burkina Faso
}

\author{
Lamine ZERBO ${ }^{1 *}, \mathrm{H}^{*}$ Bismarck NACRO ${ }^{2}$, Albert YAO-KOUAME ${ }^{3}$ et \\ P. Michel SEDOGO ${ }^{4}$
}

\author{
${ }^{1}$ Institut de l'Environnement et de Recherches Agricoles (INERA), Cellule de télédétection et de systèmes \\ d'informations Géographiques, 01 BP 476 Ouagadougou 01, Burkina Faso. \\ ${ }^{2}$ Université Polytechnique de Bobo-Dioulasso (UPB), Laboratoire d'étude et de recherche sur la fertilité du sol \\ (LERF), 01 BP 1091 Bobo-Dioulasso, Burkina Faso. \\ ${ }^{3}$ Université Felix HOUPHOUET-BOIGNY de Cocody, Laboratoire de Pédologie et de Géologie Appliquée, \\ Université Félix Houphouët-Boigny, Abidjan, 22 BP 582 Abidjan 22, Côte d'Ivoire. \\ ${ }^{4}$ Institut de l'Environnement et de Recherche Agricoles (INERA), Laboratoire sol, eau et plante, 01 BP 476 \\ Ouagadougou 01, Burkina Faso. \\ *Auteur correspondant; E-mail : zerbolamine@yahoo.fr
}

\begin{abstract}
REMERCIEMENTS
Ce travail a été réalisé avec un appui financier de l'Agence Australienne pour le Développement International (AusAID) via le Conseil Ouest et Centre Africain pour la Recherche et le développement agricoles (CORAF/WECARD) à travers le projet de recherche-développement sur les "Options d'intensification durable de l'intégration agriculture-élevage, de gestion du risque et de réduction de la vulnérabilité dans les systèmes agro-sylvo-pastoraux des zones sub-humides et semi-arides d'Afrique de l'Ouest". Nous leur témoignons toute notre gratitude.
\end{abstract}

\section{RESUME}

La dynamique des cuirasses ferrugineuses est caractérisée par un ensemble de processus, incluant leur démantèlement, le transport des altérites provenant du démantèlement et leur dépôt en aval. Ces altérites constitueront des facteurs de marginalisation des aptitudes culturales des sols sous-jacents. La présente étude a évalué les connaissances et les perceptions locales de la dynamique des cuirasses ferrugineuses des populations de deux villages du Burkina Faso. Au total, 120 personnes ont été interviewées à travers des enquêtes individuelles. Les résultats montrent que tous les producteurs interviewés connaissent la dynamique des cuirasses ferrugineuses dans leur environnement. Ces connaissances prennent en compte les preuves de la manifestation du phénomène, qui sont constituées par la présence de zones à recouvrement gravillonnaire et de ses impacts négatifs sur les activités agricoles. L'étude fait ressortir le recours par les populations, de deux types d'indicateurs pour détecter la présence en profondeur des cuirasses ferrugineuses. Il s'agit des indicateurs végétaux et des indicateurs édaphiques. L'utilisation de ces deux types indicateurs et la référence aux dépôts gravillonnaires constituent pour les producteurs, des sources de connaissance pour comprendre et expliquer la dynamique des cuirasses ferrugineuses et leur permettent d'élaborer des stratégies pour une exploitation durable de ces espaces.

(C) 2016 International Formulae Group. All rights reserved.

Mots clés : Dépôts gravillonaires, indicateurs édaphiques, indicateurs végétaux, Burkina Faso. 


\title{
Indigenous knowledge assessment of iron crust dynamic: case study in north- soudanian and south-soudanian zones of Burkina Faso
}

\begin{abstract}
The iron crust dynamic is characterized by a set of processes including its dismantling, dripping and gradual deposit of erosion materials along the landscape. These gravel deposits constitute a great problem for soils use in agriculture. This study aims to evaluate the set of indigenous knowledge of iron crust dynamic through individual investigation in two (02) villages in Burkina Faso. A sample of one hundred and twenty (120) persons has been interviewed. The results show that all of the interviewees use indigenous knowledge to describe and explain iron crust dynamic in their area. This indigenous knowledge is base, firstly, on herbaceous indicators and soils indicators, which allow the population to deduct the presence of iron crusts in depth, secondarily, the use of the gravel surface by people as material evidence of this dynamic. The study shows also that all the interviewees estimate that the iron crust dynamic has a negative impact on agricultural activities. The use of the couple of indicators added to the reference about gravels deposits are a reliable sources of knowledge which allow the local population to understand and explain the iron crust dynamics in order to elaborate some strategies for a sustainable use of these zones.
\end{abstract}

Keywords: Gravel deposits, soils indicators, herbaceous indicators, Burkina Faso.

\section{INTRODUCTION}

Les savoirs locaux et les perceptions locales constituent la base $\mathrm{du}$ processus décisionnel dans tous les domaines d'activités qui ponctuent la vie des différentes communautés locales (Dialla, 2005). Ils sont généralement transmis oralement de génération en génération et contrastent avec le système de connaissance moderne ou scientifique. Les premières études, ayant pris en compte les savoirs locaux, ont été conduites dans les années 1974 par Knight sur les pratiques agricoles des Nyiha en Tanzanie. Depuis lors, il y a eu un grand intérêt des chercheurs ainsi que des acteurs des services techniques de développement vis-à-vis des savoirs locaux et du mode de vie des populations locales. Cet élan d'intérêt a créé un domaine diversifié de savoirs avec plusieurs appellations: ethnoscience, connaissance technique endogène, savoir local (Birmingham, 2003; Dialla, 2005), ethno pédologie (Da et al., 2008; Lemoigne, 2010), ethnobotanique (Gueye et al., 2012) etc.
Quelle que soit l'appellation retenue, il est primordial de noter que ce type de savoir constitue un complément indispensable aux travaux de recherche scientifique. Selon West et al. (2008), pour mieux expliquer un phénomène au niveau d'une population donnée et assurer sa meilleure prise en charge et sa gestion, il est important de prendre en compte les connaissances et les perceptions locales sur cette problématique.

Des études sur la dynamique des cuirasses ferrugineuses (Alexandre, 2002; Temgoua et al., 2002) montrent que cette dynamique tend à marginaliser les sols soumis à son influence. Pourtant, ces sols sont de plus en plus utilisés par les populations rurales pour leurs activités de production agro-sylvopastorales. Cela suggère que dernières disposent de riches savoirs locaux pour analyser et comprendre cette dynamique et partant, qu'elles aient développé ou adopté des options de productions agro-sylvopastorales adéquates pour ces types de domaines agraires. C'est pour contribuer à 
étayer cette situation que la présente étude a été entreprise. Elle a cherché à répondre aux questions suivantes :

- quels sont les indicateurs utilisés par les populations pour certifier de la présence ou pas des cuirasses ferrugineuses, surtout en profondeur?

- ces indicateurs permettent-ils de comprendre et d'expliquer la dynamique de cette formation?

- contribuent-ils à guider au choix par les populations d'options de production agrosylvo-pastorales?

\section{MATERIEL ET METHODES}

\section{Présentation de la zone d'étude}

L'étude a été réalisée à Koumbia et à Korsimoro, deux (02) villages du Burkina Faso, situés dans deux (02) zones agroécologiques différentes. Le village de Koumbia est localisé dans la zone Sudsoudanienne tandis que celui de Korsimoro se trouve dans la zone Nord-soudanienne (Figure 1). Les coordonnées géographiques sont respectivement de $11^{\circ} 13^{\prime} 60^{\prime \prime} \mathrm{N}$ et $3^{\circ} 42^{\prime} 00^{\prime}$ ' W pour Koumbia et $12^{\circ} 49^{\prime} 24^{\prime \prime}$ N et $1^{\circ} 04^{\prime} 12^{\prime \prime}$ W pour Korsimoro. La zone Nord-soudanienne couvre la partie médiane du pays avec une pluviosité moyenne annuelle variant de 600 à $900 \mathrm{~mm}$. Elle représente $33,7 \%$ du territoire national et accueille 50\% de la population avec seulement $32 \%$ des terres arables (Ouédraogo, 2005). C'est là que se rencontrent les plus fortes densités de population du Burkina Faso. La pression foncière est très élevée à cause de la forte démographie observée. La superficie agricole par habitant est faible et varie de 0,7 à 1 hectare.

La zone Sud-soudanienne couvre la partie Sud du Burkina Faso, avec des hauteurs d'eau supérieures à $900 \mathrm{~mm}$ par an. Elle occupe $36 \%$ du territoire avec une densité moyenne de la population de 20 habitants au $\mathrm{km}^{2}$. C'est aussi la zone d'accueil de migrants en provenance d'autres régions du pays. Selon Ouédraogo 2005, l'environnement est en pleine mutation sous l'effet de la pression migratoire et des systèmes de production de type extensif. Avec moins de $30 \%$ de la population en 1985, cette zone dispose de $35 \%$ des surfaces arables du pays. Les densités de population sont relativement faibles ainsi que le coefficient d'intensité culturale (rapport entre la superficie cultivée et celle cultivable).

\section{Outils et méthodes de collecte de données de terrain}

Des entretiens individuels ont été réalisés dans les sites et ont concerné cent vingt (120) personnes soit soixante (60) personnes par site. L'échantillon est composé de quarante (40) femmes soit quinze (15) à Koumbia et vingt-cinq (25) à Korsimoro. Des chefs d'exploitation âgés de 35 ans et plus, ont été choisis au hasard pour les entretiens. Une base de données sur la typologie des ménages établie précédemment dans les deux sites d'étude par une équipe de l'Institut National de l'Environnement et de Recherches Agricoles du Burkina Faso (INERA) en 2012, a été également utilisée pour baliser certains points d'entrée: le ménage est pris comme unité d'analyse. Seul le chef de ménage est enquêté et ce dernier doit vivre dans le village depuis au moins 20 ans pour être à même de connaître le paysage et de mieux cerner les changements survenus. Les données collectées concernent les types d'indicateurs endogènes sur lesquels les populations des deux sites se basent pour déduire la présence de cuirasses ferrugineuses en profondeur. Les indicateurs de la manifestation de la dynamique des cuirasses ferrugineuses ainsi que l'influence des éléments solides issus de ce 
démantèlement sur les activités agricoles ont été également estimés.

Des travaux de terrains ont été réalisés ensuite le long des transects dans chaque village avec la participation de personnes ressources. Les noms scientifiques des espèces végétales citées lors des enquêtes paysannes ont été déterminés ainsi que leur position dans la topo-séquence et les états de surface y afférents. Ces différentes données ont été collectées à partir de fiches de terrain conçues à cet effet. Des fosses pédologiques ont été ouvertes, décrites selon les directives FAO (1994), la couleur a été déterminée à l'aide du code Munsell (1975). Ces sols ont été classés selon la Commission de Pédologie et de Cartographie des Sols (CPCS, 1967) et la corrélation avec la World Reference Base for soils ressources (WRB, 2006) a été établie. Des échantillons composites de l'horizon de surface ont été prélevés pour analyse au laboratoire du BUreau NAtional des SOLS (BUNASOLS) selon les méthodes qui y sont pratiquées (BUNASOLS, 1987).

\section{Analyse des données}

Les données collectées lors des enquêtes de terrain ont été analysées avec le logiciel SPSS version 20.0. Pour les données qualitatives (opinions de producteurs sur la dynamique des cuirasses ferrugineuses), les fréquences cumulées, exprimées en pourcentage ont été déterminées. Pour les données quantitatives (âges des producteurs, superficies et nombre des champs par producteurs, etc.) l'analyse de variance (ANOVA) a été réalisée. Il a été également procédé à la comparaison des moyennes à l'aide du test de Fisher au seuil de probabilité de $5 \%$.

Pour les échantillons de sols, les paramètres suivant ont été déterminés : granulométrie 5 fractions par le Bouyoucos hydrometer (Bouyoucos, 1927), matière organique par la méthode Walkley and Black, (Walkley et Black, 1934), azote total par la méthode Kjeldal (Hillebrand et al., 1953), phosphore assimilable par la méthode Bray I (Bray et Kurtz, 1945), potassium disponible (extraction à l'acide oxalique et chlorhydrique) et $\mathrm{pH}$ eau, par la méthode électrométrique au $\mathrm{pH}$ mètre à électrode de verre (AFNOR, 1999) .

Une analyse diachronique des surfaces gravillonnaires a été réalisée à partir de la classification supervisée des images de type Landsat sur trois dates différentes: images Landsat $\mathrm{ETM}^{+}$pour les périodes 1987 et 2000 et les images Landsat OLI pour les périodes 2013 et 2014 . Le choix des dates de prise de vue des images satellitaires a tenu compte d'une part de leur bonne qualité (absence de couverture nuageuse, etc.) et d'autre part d'une bonne visibilité de la couverture du sol. Ainsi les images de saison sèche ont été privilégiées car elles permettent de mieux distinguer les unités d'occupation des sols. Les données cartographiques ont été ensuite analysées grâce au logiciel ArcMAP version 10. Elle a consisté pratiquement à la superposition des cartes thématiques pour analyser les tendances évolutives du taux de recouvrement gravillonnaire. 

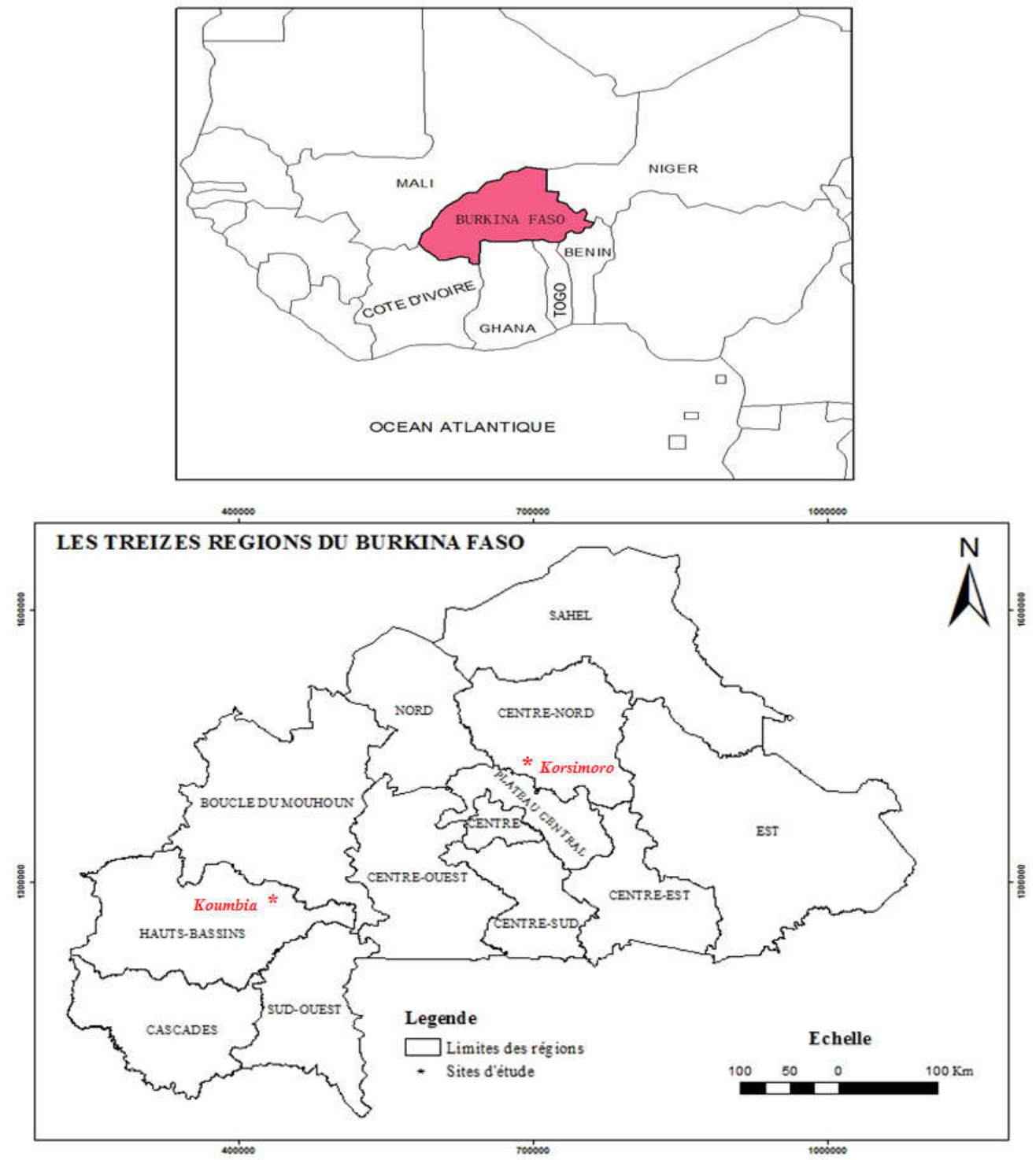

Figure 1 : Localisation du Burkina Faso et des sites d'étude.

\section{RESULTATS}

\section{Caractéristiques des exploitants}

Quelques caractéristiques des exploitants et des exploitations au niveau des deux sites sont présentées dans le Tableau 1. Quinze pourcent $(15 \%)$ des 60 producteurs enquêtés à Koumbia étaient de sexe féminin contre $25 \%$ pour le site de Korsimoro. La moyenne d'âge est de 46 ans à Koumbia et de 51 ans à Korsimoro. L'échantillon enquêté est dominé au niveau des deux sites par les autochtones, qui représentent $60 \%$ contre $40 \%$ de migrants. L'activité principale de l'échantillon enquêté des deux sites est l'agriculture qui est pratiquée par environ $95 \%$ des producteurs. L'activité secondaire est 
l'élevage qui est pratiquée par $75 \%$ des producteurs à Koumbia et par $80 \%$ des producteurs à Korsimoro. Le niveau de scolarisation de l'échantillon enquêté est de $28,3 \%$ pour les non scolarisés et $71,7 \%$ pour les scolarisés qui se répartissent comme suit : $5,4 \%$ des enquêtés ont atteint le cycle secondaire, $16,3 \%$ ont atteint le cycle primaire et $50 \%$ sont issus de l'alphabétisation non formelle. Le taux d'encadrement par les services techniques de l'Etat ou par les ONG est très important au niveau des deux sites, et est de l'ordre de $97,8 \%$.

Le nombre de parcelles par producteur est de 1 à 2 à Koumbia et de 2 à 3 à Korsimoro. Les superficies moyennes par exploitant sont plus importantes à Koumbia qu'à Korsimoro ( 8 ha et 4 ha respectivement). Les autochtones détiennent plus de parcelles et généralement de grandes superficies. L'estimation des âges des champs montre qu'il y a plus de champs récents à Koumbia qu'à Korsimoro. En effet, le nombre d'années d'exploitation des champs varie de 15 à 20 ans à Koumbia contre 20 à 25 ans à Korsimoro.

\section{Indicateurs locaux de la présence des cuirasses ferrugineuses}

Selon les résultats, $100 \%$ des producteurs enquêtés à Koumbia comme à Korsimoro reconnaissent l'existence dans leur terroir, des zones à affleurement de cuirasse ferrugineuse. Ils les localisent, selon la topographie, au niveau des collines $(80 \%)$, des cours d'eau (5\%) et des glacis moyens $(15 \%)$. Les cuirasses ferrugineuses y sont présentes sous la forme d'affleurements superficiels. Les surfaces à recouvrement gravillonnaire y sont également rencontrées. Les résultats ont montré également l'existence, dans les deux terroirs, d'indicateurs traditionnellement utilisés par les populations locales pour déduire la présence des cuirasses ferrugineuses en profondeur. Ces indicateurs peuvent être classés en deux groupes, à savoir des indicateurs édaphiques et des indicateurs végétaux.

\section{Indicateurs édaphiques}

Ces indicateurs sont en rapport avec les types de sol selon la classification locale. Les éléments morphologiques utilisés sont les suivants : - une charge graveleuse très importante en éléments grossiers, - une couleur à tendance rougeâtre, et - leur situation en hauteur dans la topo-séquence. La présence de ces trois caractéristiques au niveau d'un type de sol indique, selon tous les enquêtés, qu'il s'agit d'un sol superficiel peu profond avec comme élément limitant en profondeur, une cuirasse ferrugineuse. Le Tableau 2 donne les correspondances entre les noms locales et les noms scientifiques des sols.

A Koumbia, les producteurs ont mentionnés deux types de sol appelés en langue locale buamu «sãmbia» et «bori». Ce sont tous les deux des sols gravillonnaires, caillouteux qui se rencontrent selon $80 \%$ des enquêtés, au niveau des glacis moyens pour le premier type de sol et au niveau des collines pour le second type de sol. Les couleurs dominantes sont respectivement le rouge pour le sãmbia et le rouge-blanchâtre pour le bori.

Les profils pédologiques réalisés au niveau de sãmbia et de bori montrent que ce sont effectivement des sols peu profonds. La profondeur utile mesurée est de $18 \mathrm{~cm}$ pour le bori et de $45 \mathrm{~cm}$ pour le sãmbia. Elle est limitée par la présence d'une cuirasse ferrugineuse. La couleur, selon le code munshell est dans l'ensemble brun (10 YR 4/3) en surface mais devient rouge-jaunâtre (5 YR $5 / 8$ ou 5 YR 4/6) en profondeur, pour les deux type de sol. La charge graveleuse est importante, soit environ $60 \%$ de gravillons ferrugineux avec en plus, la présence des fragments de cuirasses ferrugineuses au niveau du bori. La texture est limoneuse, la teneur en limon est de $46,2 \%$ au niveau du bori et $50 \%$ au niveau de sãmbia. Le $\mathrm{pH}$-eau est moyennement acide au niveau des deux types de sol $(=5,75)$. Les teneurs en matière organique $(\leq 1,02 \%)$, en azote total $(=0,04 \%)$ sont faibles. Les teneurs en phosphore assimilable $\left(\leq 0,8 \mathrm{mg} \cdot \mathrm{kg}^{-1}\right)$ et en potassium 
disponible ( $\leq 20$ mg. $\mathrm{kg}^{-1}$ ) sont également faibles. Selon la CPCS (1967), le bori est un sol ferrugineux tropical lessivé induré superficiel ou leptosol lithique selon la WRB (2006) et le sãmbia correspond à un sol ferrugineux tropical lessivé induré peu profond et lixisol ferrique phase petroferrique selon la WRB (2006).

A Korsimoro, les producteurs ont également mentionnés deux types de sol en langue locale moré : zẽka et Zẽgdga. Selon les caractéristiques morphologiques, le premier est un sol latéritique faiblement gravillonnaire, tandis que le deuxième est un sol gravillonnaire, caillouteux. Dans la toposéquence, ces sols se rencontrent, selon $80 \%$ des producteurs, au niveau des collines et des glacis moyens au niveau des collines et des plateaux de glacis et selon $20 \%$ des enquêtés, en bas de pente des glacis moyens.

Les profils pédologiques réalisés au niveau de zẽka et de Zẽgdga sont également des sols peu profonds. La profondeur utile est de $20 \mathrm{~cm}$ pour le Zẽgdga et de $50 \mathrm{~cm}$ pour le $z e \tilde{k} a$. Ils sont limités en profondeur par une cuirasse ferrugineuse. La couleur, selon le code munsell, est dans l'ensemble brun (10 YR 4/4) en surface et jaune-rougeâtre (7,5 YR $6 / 8$ ) en profondeur. La texture est limosableuse, la teneur en sable est très importante, soit de l'ordre de $67 \%$. La charge graveleuse est plus importante au niveau du Zẽgdga $(50-60 \%)$ qu'au niveau du zẽka $(40 \%)$. Le pH-eau est dans l'ensemble moyennement acide $(=5,73)$. Leurs teneurs en matière organique $(\leq 1,18 \%)$ et en azote total $(\leq 0,04 \%)$ sont faibles. Les teneurs en phosphore assimilable $\left(\leq 0,7 \mathrm{mg} \cdot \mathrm{kg}^{-1}\right)$ et en potassium disponible $\left(\leq 54 \mathrm{mg} \cdot \mathrm{kg}^{-1}\right)$ sont également faibles. Selon la CPCS (1967), le Zẽgdga correspond à un sol ferrugineux tropical lessivé induré superficiel et leptosol lithique selon la WRB (2006); tandis que le $z e \tilde{k} a$ est un sol ferrugineux tropical lessivé induré peu profond et lixisol ferrique phase petroferrique selon la WRB (2006).

\section{Indicateur végétal}

Les indicateurs principaux de ce groupe sont des herbacés de type graminéen. Selon les producteurs, la présence de ces herbacées, en grand nombre, au niveau d'une zone donnée, peut indiquer la présence d'une cuirasse ferrugineuse en position «peu profonde». Ainsi à Koumbia, tous les producteurs enquêtés ont mentionné deux herbacées, il s'agit de sani et sapore en buamu. Ces herbacés ont été mentionnées respectivement par $70 \%$ et par $30 \%$ des enquêtés. A Korsimoro, $100 \%$ des producteurs enquêtés ont cité aussi deux herbacés, il s'agit en langue locale moore de sũtu (citée par $90 \%$ des enquêtés) et de na-tũ-n-kuili (mentionnée par $10 \%$ des enquêtés). Selon l'ensemble des enquêtés des deux sites, si ces herbacées sont rencontrées sur des sols gravillonnaires, la certitude d'y trouver la cuirasse ferrugineuse en profondeur est plus grande. Les travaux de terrain ont permis de déterminer les noms scientifiques des espèces citées ainsi que les types de sol sur lesquels elles ont été rencontrées (Tableau 3). Du point de vue botanique, il s'agit de Eragrostis tremula Steud. (sani) et de Digitaria horizontalis Willd. (sapore) dans le site de Koumbia. Elles appartiennent à la famille des Poaceae. A Korsimoro, il s'agit de Loudetia togoensis (Pilg.) C. E. Hubb. (sũtu) et de Zornia glochidiata Rchb. Ex DC. (na-tũ-n-kuili). La première citée appartient à la famille des Poaceae tandis que la seconde fait partie de la famille des Fabaceae. Du point de vue pédologique, les grandes concentrations de ces herbacées ont été rencontrées essentiellement sur des sols gravillonnaires. Ainsi, à Koumbia, Eragrostis tremula Steud. et Digitaria horizontalis Willd. ont été rencontrés à plus de $90 \%$ au niveau des sols gravillonnaires situés sur les glacis moyens. A Korsimoro la situation est plus mitigée pour Zornia glochidiata Rchb. Ex DC. En effet, si Loudetia togoensis (Pilg.) C. E. Hubb. se rencontre à plus de $90 \%$ sur les sols gravillonnaires des glacis moyens et au niveau des collines, le Zornia glochidiata Rchb. Ex DC. a été plus rencontrée au niveau des sols 
limono-sableux situés au niveau de zones de dépressions où stagne temporairement l'eau de ruissellement. Le profil pédologique qui a été réalisé montre un sol peu profond, la profondeur utile est de $62 \mathrm{~cm}$, mais avec une faible teneur en éléments grossiers. Il est limité par une cuirasse ferrugineuse. La couleur, selon le code munsell, est dans l'ensemble gris clair (10 YR 7/2) en surface et jaune-rougeâtre $(7,5$ YR 6/6) en profondeur. La texture est limono-sableuse en surface et limono-argileuse en profondeur. La charge graveleuse est importante en profondeur, soit de l'ordre de $40 \%$. Le pH-eau est dans l'ensemble moyennement acide $(=5,60)$. Leurs teneurs en matière organique $(=1,21 \%)$ et en azote total $(=0,06 \%)$ sont légèrement moyennes. Les teneurs en phosphore assimilable $\left(=0,86 \mathrm{mg} \cdot \mathrm{kg}^{-1}\right)$ et en potassium disponible $\left(=65 \quad \mathrm{mg} \cdot \mathrm{kg}^{-1}\right)$ sont également faibles. Il s'agit d'un sol ferrugineux tropical lessivé induré peu profond (CPCS, 1967) et lixisol ferrique phase petroferrique (WRB, 2006).

\section{Perception locale de la dynamique des surfaces cuirassées \\ De l'avis de l'ensemble des} producteurs enquêtés dans les deux sites, le démantèlement des cuirasses ferrugineuses est une réalité dans leur terroir. Il se traduit concrètement sur le terrain par la présence d'éléments solides qu'ils appellent «gravillons» comprenant des gravillons ferrugineux et des concrétions ferrugineuses. Cependant, l'ampleur de ces dépôts n'est pas la même dans les deux terroirs. A Koumbia, dans la zone Sud-soudanienne, $16 \%$ des producteurs enquêtés trouvent le démantèlement des cuirasses ferrugineuses très important, $60 \%$ le trouvent peu important et $24 \%$ le considèrent insignifiant. A Korsimoro, dans la zone Nord-soudanienne, $62 \%$ des producteurs enquêtés le trouvent très important, $25 \%$ le trouvent peu important et $13 \%$ le considèrent insignifiant. L'ensemble des producteurs enquêtés affirment donc que le phénomène est fréquent à très fréquent sur les flancs des collines, au niveau des versants des plateaux cuirassés et au niveau de certains cours d'eau. Le pas de temps pour la perception de cette dynamique est estimé à environ 15 à 20 ans selon les enquêtés. Pour les autres zones de la toposéquence, l'épandage des produits gravillonnaires issus du démantèlement de la cuirasse ferrugineuse y est rare. En effet, plus de $80 \%$ des producteurs enquêtés des deux terroirs disent que l'épandage gravillonnaire n'y est pas visible actuellement, mais pourrait être perceptible à très long terme. Ainsi, $90 \%$ des producteurs enquêtés à Koumbia contre $75 \%$ à Korsimoro, donnent un pas de temps d'environ 30 ans pour que cet épandage soit perceptible dans ces zones. Dans les deux sites, $92 \%$ des producteurs enquêtés reconnaissent l'impact négatif des produits solides issus du démantèlement de la cuirasse ferrugineuse sur les activités agricoles. Les paramètres impactés les plus fréquemment cités sont, par ordre d'importance : la perte de la fertilité des sols, le travail difficile de ces types de sols et le faible enracinement des cultures pouvant entrainer leur chute en cas de vent violent.

Les résultats de l'analyse diachronique (Figure 2) des surfaces à recouvrement gravillonnaire devrait permettre d'expliquer leurs tendances évolutives au cours des vingt-sept (27) années écoulées (1987 à 2014). A Koumbia, les zones à recouvrement gravillonnaire par rapport à la superficie totale représentent $14,40 \%$ en 2014 , contre $8,60 \%$ en 2000 et $8,50 \%$ en 1987 , donc une progression perceptible de ces zones au cours des années. Cette progression est de l'ordre de $0,1 \%$ entre 1987 et 2000 et de 5,4\% entre 2000 et 2014. A Korsimoro, les zones à recouvrement gravillonnaire par rapport à la superficie totale représentent $38,28 \%$ en 1987 , $43,6 \%$ en 2000 et $53,3 \%$ en 2013. La progression est plus marquée ici. En effet de $5,32 \%$ entre 1987 et 2000 , elle passe à $9,70 \%$ entre 2000 et 2013. 
Tableau 1 : Quelques caractéristiques des exploitations étudiées.

\begin{tabular}{|c|c|c|c|}
\hline & & Site de Koumbia & Site de Korsimoro \\
\hline \multirow{3}{*}{$\begin{array}{l}\text { Nombre total } \\
\text { personnes enquêtées }\end{array}$} & Femmes & 15 & 25 \\
\hline & Hommes & 85 & 75 \\
\hline & Total & 100 & 100 \\
\hline \multicolumn{2}{|l|}{ Age moyen (années) } & 46 & 51 \\
\hline \multirow{2}{*}{$\begin{array}{lll}\text { Origine des } & \text { personnes } \\
\text { enquêtées }(\%) & \end{array}$} & Autochtones & 60 & 60 \\
\hline & Migrants & 40 & 40 \\
\hline \multicolumn{2}{|c|}{ Nombre de champs/exploitant (années) } & $1-2$ & $2-3$ \\
\hline \multicolumn{2}{|l|}{ Superficie par exploitant (ha) } & 08 & 4 \\
\hline \multicolumn{2}{|c|}{ Age moyen de champ (années) } & $15-20$ & $20-25$ \\
\hline \multicolumn{2}{|c|}{ Couverture de l'encadrement technique (\%) } & 97,8 & 97,8 \\
\hline
\end{tabular}

Tableau 2 : Correspondances des noms locales et des noms scientifiques des sols.

\begin{tabular}{|c|c|c|c|}
\hline & $\begin{array}{l}\text { Noms } \\
\text { locales }\end{array}$ & Noms selon CPCS (1967) & Noms selon WRB (2006) \\
\hline \multirow[b]{2}{*}{ Koumbia } & Sãmbia & $\begin{array}{l}\text { Sols ferrugineux tropicaux lessivés } \\
\text { indurés peu profonds }\end{array}$ & $\begin{array}{l}\text { Lixisol ferrique } \\
\text { petroferrique }\end{array}$ \\
\hline & Bori & $\begin{array}{l}\text { Sols ferrugineux tropicaux lessivés } \\
\text { indurés superficiels }\end{array}$ & Leptosol lithique \\
\hline \multirow{2}{*}{ Korsimoro } & Zêka & $\begin{array}{l}\text { Sols ferrugineux tropicaux lessivés } \\
\text { indurés peu profonds }\end{array}$ & $\begin{array}{l}\text { Lixisol ferrique phase } \\
\text { petroferrique }\end{array}$ \\
\hline & Zẽgdga & $\begin{array}{l}\text { Sols ferrugineux tropicaux lessivés } \\
\text { indurés superficiels }\end{array}$ & Leptosol lithique \\
\hline
\end{tabular}


Tableau 3 : Indicateurs locaux de la présence des cuirasses ferrugineuses.

\begin{tabular}{|c|c|c|c|c|c|c|}
\hline & \multicolumn{3}{|c|}{ Koumbia } & \multicolumn{3}{|c|}{ Korsimoro } \\
\hline & $\begin{array}{l}\text { Appellation } \\
\text { locale }\end{array}$ & $\begin{array}{c}\text { Nom } \\
\text { scientifique }\end{array}$ & $\begin{array}{c}\text { Caractéristiques } \\
\text { de la surface des } \\
\text { sols }\end{array}$ & $\begin{array}{l}\text { Appellation } \\
\text { locale }\end{array}$ & $\begin{array}{c}\text { Nom } \\
\text { scientifique }\end{array}$ & $\begin{array}{c}\text { Caractéristiques } \\
\text { de la surface des } \\
\text { sols }\end{array}$ \\
\hline \multirow{2}{*}{$\begin{array}{l}\text { Indicateur } \\
\text { végétal }\end{array}$} & sani & $\begin{array}{l}\text { Eragrostis } \\
\text { tremula }\end{array}$ & $\begin{array}{l}\text { Recouvrement } \\
\text { gravillonnaires }\end{array}$ & Sũtu & $\begin{array}{l}\text { Loudetia } \\
\text { togoensi }\end{array}$ & $\begin{array}{l}\text { Recouvrement } \\
\text { gravillonnaire }\end{array}$ \\
\hline & sapore & $\begin{array}{l}\text { Digitaria } \\
\text { Horizontalis }\end{array}$ & $\begin{array}{l}\text { Recouvrement } \\
\text { gravillonnaires }\end{array}$ & $n a-t \tilde{u}-n-k u i l i$ & $\begin{array}{l}\text { Zornia } \\
\text { glochidiata }\end{array}$ & $\begin{array}{l}\text { Recouvrement } \\
\text { limono-sableux }\end{array}$ \\
\hline
\end{tabular}

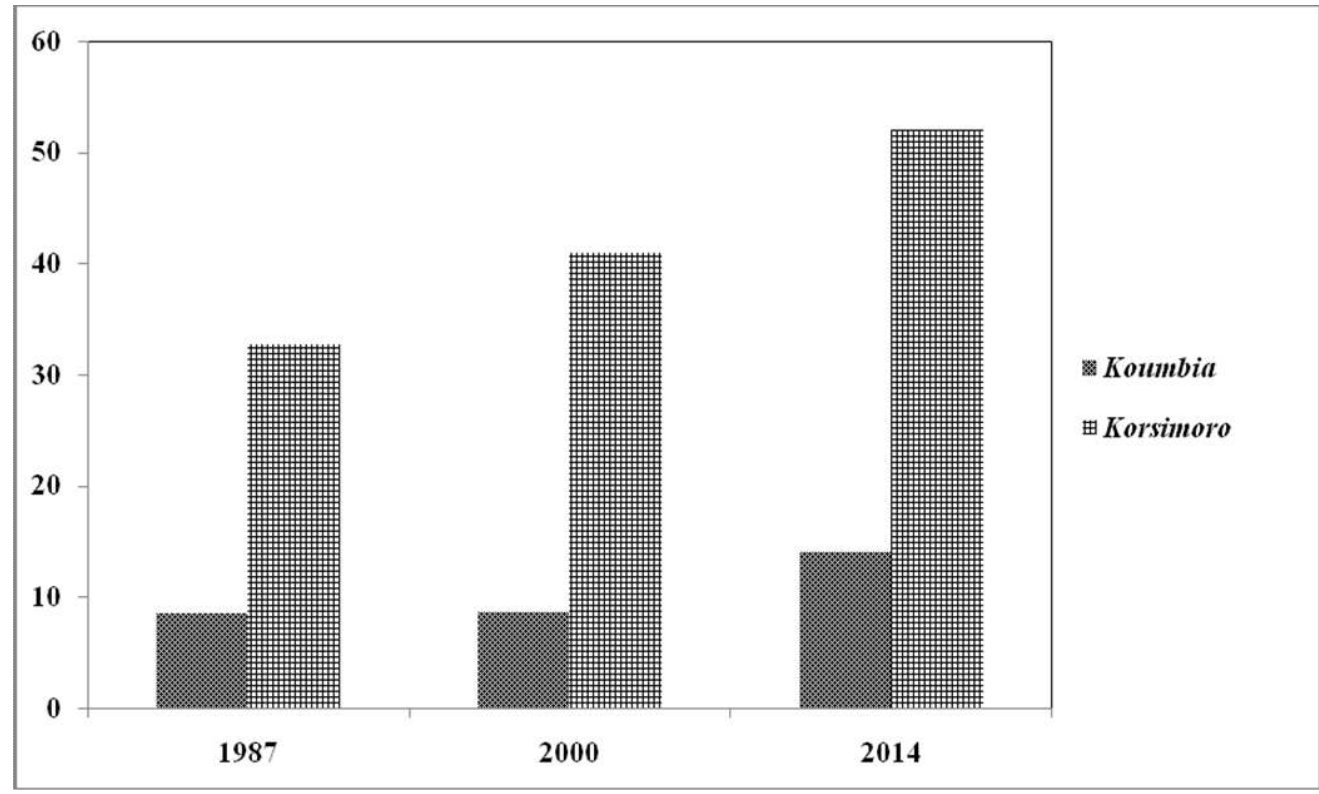

Figure 2 : Tendances évolutives des surfaces à recouvrement de gravillons dans les deux sites.

\section{DISCUSSION}

Indicateurs locaux de la présence des cuirasses ferrugineuses

Les indicateurs utilisés par les producteurs pour déduire la présence des cuirasses ferrugineuses sont de deux types: les indicateurs édaphiques et les indicateurs végétaux. L'ensemble des indicateurs édaphiques cités par les producteurs des deux sites correspondent à des unités de sol selon leur classification traditionnelle des sols. Il s'agit de sãmbia et de bori à Koumbia, de $z \tilde{e} k a$ et de Zẽgdga à Korsimoro. Les critères utilisés par les populations pour leur distinction sont: une charge graveleuse caractérisée par un recouvrement gravillonnaire moyen ou élevé et une situation topographique sur les hauts de pente et sur les mi-pentes. La description et la classification selon la WRB (2006), des fosses pédologiques 
qui y ont été réalisées ont révélées qu'il s'agit de leptosols lithiques (bori et Zẽgdga) et de lixisols ferriques phase petroferrique (sãmbia et $z e \tilde{k} a$ ). Selon la WRB (2006), ces deux types de sols sont caractérisés par des critères diagnostics principaux suivants : la présence de gravillons ferrugineux et la présence d'une couche limitante en profondeur constituée d'une roche dure continue. L'ensemble de ces constats est confirmé par les travaux de Dialla (2005), Vall et Diallo (2009), Kissou et al. (2012), faisant de l'utilisation de cet indicateur par les populations locales, une source de connaissance assez fiable pour déduire de la présence en profondeur d'une cuirasse ferrugineuse.

Les indicateurs végétaux sont caractérisés de bio-indicateurs par des études antérieures (Chotte et al., 2001; IlboudoTapsoba et al., 2011; Ogni et al., 2014) car ils sont utilisés par les populations locales comme indicateur de l'état de fertilité des sols. Il s'agit de Eragrostis tremula Steud. (sani) et de Digitaria horizontalis Willd. (sapore) à Koumbia, Loudetia togoensis (Pilg.) C. E. Hubb. (sũtu) et de Zornia glochidiata Rchb. Ex DC. (na-tũ-n-kuili) à Korsimoro. Selon Achard et al. (2001), ces herbacées se rencontrent fréquemment dans les régions sahélo-soudaniennes et soudaniennes, avec une pluviosité annuelle comprise entre 600 et $1400 \mathrm{~mm}$ comme c'est le cas à Koumbia et à Korsimoro où la pluviométrie moyenne annuelle de 2000 à 2013 est respectivement de $956 \mathrm{~mm}$ et de 633 $\mathrm{mm}$. Selon les mêmes auteurs, ces bioindicateurs sont des espèces végétales caractéristiques des sols peu profonds, dégradés avec un paramètre limitant en profondeur sous la forme de cuirasse ferrugineuse ou de roches diverses. Ces conclusions concordent avec les résultats des études pédologiques réalisées dans les deux sites et qui montrent la présence de cuirasse ferrugineuse en profondeur au niveau des sols portant ces indicateurs végétaux. $\mathrm{La}$ différence des espèces végétales citées dans les deux terroirs, peut être liée aux conditions à la fois agro-climatique et socio-économique qui favoriseraient le développement et la prolifération de certaines espèces dans une zone donnée au détriment d'autres espèces.

\section{Démantèlement des cuirasses ferrugineuses et impacts sur les sols}

Le démantèlement des cuirasses ferrugineuses est une réalité dans les deux terroirs selon les résultats d'enquêtes. Les preuves données par les producteurs sont constituées d'un ensemble de zones portant des altérites de cette dégradation. Ces constats sont confirmés par les résultats des travaux antérieurs (Alexandre, 2002). Le démantèlement des cuirasses ferrugineuses se fait essentiellement sous les actions conjuguées des agents anthropiques, mécaniques et biologiques. Les altérites issues de ce démantèlement sont essentiellement des gravillons ferrugineux et des concrétions ferrugineuses; et forment des zones homogènes plus ou moins étendues (Temgoua et al., 2002). On peut y ajouter également des zones de pavage de cailloux et de pierres laissés en surface, une fois que les particules les plus fines du sol sont emportées par le ruissellement (Kabré, 2009). De l'avis des producteurs, ces zones de dépôt sont rencontrées fréquemment à très fréquemment, au niveau les flancs des collines et dans certains bas-fonds. Ces zones de dépôt sont rares au niveau des versants. Ces résultats corroborent ceux de Temgoua et al. (2002) qui utilisent les termes techniques de géomorphologie à savoir : haut de pente, mipente et bas de pente et qui correspondent respectivement aux flancs des collines, aux versants et à l'ensemble constitué par les berges et les bas-fonds. Les analyses ont montré que l'ampleur des dépôts n'est pas le même selon les terroirs, il est plus important à Korsimoro qu'à Koumbia. Ce constat est confirmé par l'analyse diachronique des surfaces gravillonnaires réalisée dans les deux sites. Selon des études antérieures (Alexandre, 
2002; Temgoua et al., 2002), cette différence serait liée à plusieurs facteurs qui influencent le processus de démantèlement et d'épandage des altérites. Il s'agit notamment de la couverture végétale, de la structure des cuirasses ferrugineuses, de l'activité biologique et du processus de transport et de dépôt des altérites. Ces facteurs sont susceptibles de freiner ou de favoriser les processus de démantèlement, de transport et de dépôt des altérites.

L'impact négatif de l'ensemble de ces altérites sur l'utilisation agricole des terres concernées est perçu par environ $90 \%$ des producteurs enquêtés sur les deux sites. Ces résultats sont confirmés par plusieurs travaux de recherche (Ouédraogo et al., 2001; Savadogo et al., 2011). Les paramètres qui sont les plus impactés par ce processus et qui sont les plus cités sont par ordre d'importance: la fertilité des sols qui subit une baisse, la dureté des sols qui les rend très difficiles à travailler, le faible enracinement des cultures au niveau de ces sols pouvant entrainer leur chute en cas de vent violent. La mise en valeur de ces types de sol impose donc aux populations concernées, le développement et/ou l'adoption d'un ensemble de comportements et de technologies adaptées.

\section{Conclusion}

L'objectif de l'étude était d'évaluer les connaissances et les perceptions locales de la dynamique des cuirasses ferrugineuses par les populations de deux villages du Burkina Faso. Il s'agissait également de déterminer leur contribution au choix de stratégies pour une exploitation durable de ces espaces instables par les populations. L'étude a montré que les populations des deux sites utilisent deux types d'indicateurs pour déduire la présence en profondeur des cuirasses ferrugineuses dans une zone donnée. Il s'agit des indicateurs végétaux et édaphiques. Les indicateurs végétaux sont des plantes herbacées annuelles, tandis que les indicateurs édaphiques font référence à la teneur en éléments grossiers et à la position topographique. Pour l'estimation de la dynamique des cuirasses ferrugineuses, les populations locales se basent sur la présence des altérites grossières issues de leur désagrégation. L'étude a en outre permis de montrer que l'utilisation de ces deux types indicateurs par les populations locales, constitue des sources de connaissance fiables pour comprendre et expliquer la dynamique des cuirasses ferrugineuses et aussi pour déduire de la présence en profondeur d'une cuirasse ferrugineuse. La teneur en éléments grossiers ainsi que les informations sur la présence d'une cuirasse ferrugineuse en profondeur, sont des facteurs très importants à prendre en compte pour l'évaluation des aptitudes agro-sylvo-pastorales des terres. Ainsi, partant de leurs connaissances et perceptions, les populations locales ont élaboré des stratégies pour une exploitation durable de ces espaces: utilisation des cordons pierreux, affectation de spéculations adaptées à ces types de sols, utilisation des techniques culturales appropriées, etc... La présente étude est une contribution à la formulation de recommandations à l'endroit des producteurs et des décideurs politiques pour la gestion efficiente des terres agricoles. Ces résultats peuvent également servir de base à la mise en ouvre d'autres activités de recherche pour mieux approfondir des aspects non abordés ou insuffisamment abordés dans cette étude.

\section{CONFLIT D'INTERETS}

L'ensemble des auteurs affirment à l'unanimité l'absence de conflit d'intérêt.

\section{CONTRIBUTIONS DES AUTEURS}

LZ a collecté les données sur le terrain, il a réalisé les analyses statistiques et a écrit le manuscrit $(50 \%)$. HBN a contribué à l'amélioration de l'approche méthodologie. Il a également corrigé le manuscrit (30\%). AYK et PMS ont contribué à l'amélioration de l'approche méthodologique et ont apporté 
leurs conseils pendant la rédaction $\mathrm{du}$ manuscrit ( $10 \%$ chacun).

\section{REMERCIEMENTS}

Les images Landsat ETM + et OLI ont été téléchargées gratuitement sur le site web de l'USGS (http://landsat.usgs.gov/). Nous exprimons nos remerciements aux populations des sites de Koumbia et de Korsimoro pour leur disponibilité et leur appui au cours de nos travaux de terrain.

\section{REFERENCES}

Achard F, Hiernaux P, Banoin M. 2001. La Jachère en Afrique Tropicale, Floret et Pontanier (eds). John Libbey Eurotext: Paris; 201-239.

AFNOR. 1999. Détermination du pH. (Association Française de Normalisation) NF ISO103 90, AFNOR Qualité des sols, Paris; 339-348.

Alexandre J. 2002. Les cuirasses latéritiques et autres formations ferrugineuses tropicales exemple du haut Katanga méridional. Musée royal de l'Afrique centrale - Tervuren, Belgique. Annales Sciences Géologiques, 107: 118 p.

Birmingham DM. 2003. Local knowledge of soils: the case of contrast in Côte d'Ivoire. Geoderma, 111: 481-502.

Bouyoucos GJ. 1927. Directions for determining the colloidal materiel of soil by hydrometer method. Science Magazine, 66(1696): 16-17.

Bray RH, Kurtz LT. 1945. Determination of total organic and available forms of phosphorous in soils. Soil Science, 59: $39-45$.

BUNASOLS, 1987. Méthodes d'analyse physique et chimique des sols, eaux et plantes. Documents technique $\mathrm{n}^{\circ} 3$. Ouagadougou, 159 p. + Annexes.

Chotte JL, Duponnois R, Cadet P, Adiko A, Villenave C, Agboba C, Brauman A. 2001. Jachère et biologie du sol en Afrique tropicale. In La Jachère en Afrique Tropicale. De la Jachère Naturelle à la Jachère Améliorée, Floret
C, Pontanier R (eds). John Libbey.

Eurotext : Paris ; 85-121.

CPCS. 1967. Classification des Sols. Publ. ENSA-GRIGNON: France ; 96 p.

Da DEC, Yacouba H, Yonkeu S. 2008. Unités morphopédologiques et gestion de la fertilité des sols dans le Centre-Nord du Burkina Faso par les populations locales. Int. J. Biol. Chem. Sci., 2(3): 306-315.

Dialla BE. 2005. Pratiques et savoirs paysans au Burkina Faso. Une présentation de quelques études. Série document de travail, DT-CAPES $\mathrm{N}^{\circ}$ 2005-20, Ouagadougou, Burkina Faso, 25 p.

FAO - ISRIC 1994. Directives pour la description des sols $\left(3^{\text {éme }}\right.$ édition, révisée), Service des sols-ressources, aménagement et conservation, Division de la mise en valeur des terres et des eaux, $78 \mathrm{p}$.

Gueye M, Cissé A, Diatta CD, Diop S, Koma S. 2012. Etude ethnobotanique des plantes utilisées contre la constipation chez les Malinké de la communauté rurale de Tomboronkoto, Kédougou (Sénégal). Int. J. Biol. Chem. Sci., 6(2): 773-781.

Hillebrand WF, Lundell GEF, Bright HA, Hoffman JI. 1953. Applied Inorganic Analysis $\left(2^{\text {nd }}\right.$ edn $)$. John Wiley, Sons, Inc: New York, USA; p. 1034.

Ilboudo-Tapsoba E, Tankoano H, Ouedraogo M, Dicko IO, Sanon A. 2011. Diversité des insectes actifs au sol dans quatre écosystèmes de bas-fonds du Burkina Faso : importance pour la détermination de bio-indicateurs caractérisant ces milieux. Int. J. Biol. Chem. Sci., 5(2): 724-738.

Kabré M. 2009. Vulnérabilité des sols à l'érosion dans la région du centre nord $\mathrm{du}$ Burkina Faso: approche par télédétection et SIG, DESS/RECTAS, Université d'Obafemi Awolowo, Nigeria, $51 \mathrm{p}$.

Kissou R, Gnankambary Z, Nacro HB, Thiombiano L, Sourabié IN, Zombré NP, Sédogo PM. 2012. Corrélation, 
dénomination et perception endogènes de la fertilité des sols dans la zone centre-nord du Burkina Faso. Science et Technique, série Sciences Naturelles et Agronomie, 32(1-2): 33- 45.

Lemoigne N., 2010. Mémoire des hommes, mémoire des sols. Étude ethnopédologique des usages paysans du Mont Cameroun. Thèse de Doctorat de géographie, Université de Bordeaux III, Bordeaux, $430 \mathrm{p}$.

Munsell code 1975. Soil color charts, 34 p.

Ogni CA, Kpodekon MT, Dassou HG, Boko CK, Koutinhouin BG, Dougnon JT, Youssao AKI, Yedomonhan $\mathrm{H}$, Akoegninou A. 2014. Inventaire ethnopharmacologique des plantes utilisées dans le traitement des pathologies parasitaires dans les élevages extensifs et semi-intensifs du Bénin. Int. J. Biol. Chem. Sci., 8(3): 1089-1102.

Ouédraogo E, Mando A, Zombré NP. 2001. Use of compost to improve soil properties and crop productivity under low input agricultural system in West Africa. Agriculture, Ecosystems \& Environment, 84(3): 259-266.

Ouédraogo S. 2005. Intensification de l'agriculture dans le plateau central du Burkina Faso: Une Analyse des possibilités à partir des nouvelles technologies. Thèse de Ph.D, Centre d'étude pour le développement, Université de Groningen, Pays-Bas, $322 \mathrm{p}$.

Savadogo M, Somda J, Seynou O, Zabré S, Nianogo AJ. 2011. Catalogue des bonnes pratiques d'adaptation aux risques climatiques au Burkina Faso. UICN Burkina Faso: Ouagadougou, Burkina Faso; $52 \mathrm{p}$.

Temgoua E, Bitom D, Bilong P, Lucas Y, Pfeifer HR. 2002. Démantèlement des paysages cuirassés anciens en zones forestières tropicales de l'Afrique centrale: formation d'accumulations ferrugineuses actuelles en bas de versants. C. R. Geosciences, 334(8): 537-543.

Vall E, Diallo MA. 2009. Savoirs techniques locaux et pratiques : la conduite des troupeaux aux pâturages (Ouest du Burkina Faso). Nat. Sci. Soc., 17(2): 122-135.

Walkley A, Black RN. 1934. An examination of the method Dedtjareff for determining soil organic matter and to proposed modification of the chromic acid titration method. Soil Science, 37: 29-38.

West CT, Roncoli C, Ouattara F. 2008. Local perceptions and regional climate trends on the Central Plateau of Burkina Faso. Land Degradation \& Development, 19(3): 289-304.

WRB. 2006. World reference base for soil resources. A framework for international classification, correlation and communication, world soil resources reports $103,128 \mathrm{p}$. 